\title{
Up Close and Personal
}

\author{
Neil H. Winawer, MD, SFHM \\ Emory University School of Medicine, Atlanta, GA, USA.
}

KEYWORDS: patient-physician relationship; diagnosis; observation.

$J$ Gen Intern Med 25(11):1255-6

DOI: $10.1007 / \mathrm{s} 11606-010-1462-2$

() Society of General Internal Medicine 2010

A fter signing out the last patient in the medical clinic of our downtown teaching hospital, my exhausting day had finally come to an end. I drew a sigh of relief and relaxed in the serenity of what hours ago was a chaotic scene of patients and providers. My colleagues were long since gone. The heavy metal gate at the reception desk had been lowered and locked, the lights were dimmed, and a housekeeper industriously emptied small wastebaskets into a larger trashcan.

Before leaving, I took one final stroll through the clinic, craning my neck down each corridor in hope of spotting one of the remaining nurses. I knew a quick wave and an "everything okay?" was a good way to avoid any unforeseen problems, and equally as important, remain in good graces with the staff.

Finally, I located the nurse standing in the opposite hall. From a distance, I could make out a pained expression on her face. As I approached, she sighed and then spoke. "I don't know how we missed him, Dr. Winawer, but there's someone still out there. I tried to ask him to reschedule, but he wasn't really too happy about that. I know it's late, but can you just talk to him?"

I clenched my teeth in frustration and paused. "Sure," I offered, and headed out to the waiting room.

The clinic's lone occupant, Mr. Clark, reached out, shook my hand and then proceeded to rant about the clinic's poor scheduling practices. I tried hard not to interrupt, willing myself to be patient. Eventually he got around to telling me what was wrong.

"I just feel exhausted, Doc. Maybe my thyroid medicine needs increasing?"

"Any weight gain?"

"No, dropped over twenty. Can't seem to keep it on."

I looked him over carefully as he spoke-eyeballing his thyroid gland, inspecting for temporal wasting. Nothing was leaping out at me; Mr. Clark looked perfectly fine. I considered documenting his complaint and suggesting he reschedule soon, but the weight loss just didn't make sense.

Published online August 10, 2010
"Why don't we talk some more inside?" I asked, gesturing to the nearest exam room. While asking more questions, I checked his vital signs-all of which were normal. I quickly ran my hands up and down his neck for lymphadenopathy-again, nothing unusual. "Do you mind lifting your shirt?" I queried while reaching for my stethoscope. While I had no expectation of an auscultatory breakthrough, at least the patient couldn't say the doctor hadn't given him a "once over." I leaned in with my stethoscope and squinted my eyes. As suspected, there was nothing remarkable-but just as I removed my instrument from his skin, I noticed something. I didn't hear, or see, or even palpate that something; I smelled it. A fleeting trace of what smelled like nail polish remover crossed my nares and was gone in an instant.

"Have you been peeing a lot sir?" I questioned.

"All the time, Doctor."

"How about drinking? Have you been drinking more than normal?"

He pointed to a 2-L sports drink and large bottle of water tucked away in his backpack. I nodded my head and continued.

"Blurry vision?"

"Yes," he answered, gazing at me as if I were some sort

of psychic. He repeated for emphasis, "Yes."

I left the room and bargained with a departing nurse technician for one last fingerstick. Mr. Clark's stable appearance certainly didn't make her any more sympathetic, but soon enough she slid the strip into the glucometer and let out an audible gasp. The LCD screen flashed a "HIGH HIGH HIGH"code for $>500 \mathrm{mg} / \mathrm{dl}$.

In the interest of time, I quickly thanked the nurse and offered to walk the patient down to the emergency center triage. Upon arrival, I directed him to the intake nurse, gave her a quick synopsis and bid him farewell. Now my day was finally over.

The next morning, I entered the hospital wondering what had become of Mr. Clark. I pulled up his initial labs and stared at the screen in disbelief; his blood glucose in the emergency room was $1,358 \mathrm{mg} / \mathrm{dl}$ ! I made a beeline for his room and found him resting comfortably in a tightly monitored step down unit, flanked by his medical team. They had already heard the story of the mystery doctor who had smelled something "funny" in the clinic. While the medical students seemed impressed, all I could think about was how close I had been to sending a critically ill patient home. 
From antiquity to the present, physicians have always relied on their senses in diagnosing and treating disease. In ancient Egypt and Mesopotamia, the earliest physicians made diagnoses and recommended treatments based primarily on observation of clinical symptoms. The ancient Greeks took it even further, relying on auscultation, palpation and the importance of visualizing bodily fluids to help them predict disease. Hippocrates advocated a diagnostic protocol that included tasting the patient's urine, listening to the lungs, and observing skin color and other outward appearances. While sampling bodily fluids is thankfully no longer considered standard medical practice, the use of sight, sound, touch and olfaction remain every bit as important in medicine today as they were thousands of years ago.
In addition to reinforcing the importance of honing these physical senses, my experience highlights the necessity of taking a closer look when clinical uncertainty exists. Whether due to time constraints or the ability to order any conceivable test, modern medicine unwittingly obliges physicians to keep patients at arm's length. However, as caring for Mr. Clark confirmed, in order to detect certain subtleties on physical examination-or other interpersonal nuances germane to the care we provide-we must first get up close and personal with our patients.

Corresponding Author: Neil H. Winawer, MD, SFHM, Emory University School of Medicine, 49 Jessie Hill Jr. Dr. SE, Atlanta, GA 30303, USA (e-mail: nwinawe@emory.edu). 